Aufsatz

Rainer Meyer zum Felde*

\title{
Abschreckung und Dialogbereitschaft - der Paradigmenwechsel der NATO seit 2014
}

https://doi.org/10.1515/sirius-2018-2001

Kurzfassung: Die NATO war für zwei Jahrzehnte primär eine politische Allianz, die Russland als Partnerstaat ansah. Mit der Annexion der Krim durch Russland sowie der hybriden Aggression gegen die Ost-Ukraine hat sich ein Paradigmenwechsel vollzogen, bei dem es darum geht, einerseits Verteidigungsfähigkeit herzustellen, andererseits aber auch die Dialogbereitschaft zu erhalten. Der Beitrag zeichnet den Weg der NATO seither nach und befasst sich mit der Frage, was der Paradigmenwechsel der NATO - an dessen Zustandekommen die Bundesregierung maßgebend beteiligt war - für die deutsche Sicherheitspolitik und die Bundeswehr bedeutet. Dabei wird vor allem auf die Zusagen Deutschlands eingegangen, ambitionierte NATO-Fähigkeitenziele zu erfüllen und das Verteidigungsinvestitionsversprechen von Wales einzuhalten. Außerdem wird Deutschlands Rolle als Rahmennation behandelt. Abschließend werden weitergehende Perspektiven einer wieder stärker an Abschreckung und Bündnisverteidigung orientierten NATO aufgezeigt.

Schlüsselwörter: NATO, Verteidigungsstrategie, Abschreckung, Deutschland

Abstract: The North Atlantic Treaty Organisation has conceived of Russia as a partner state for more than two decades. This has changed with the Russian occupation and annexation of the Crimean Peninsula in March 2014 and the subsequent hybrid aggression against the Eastern Ukraine. Since that, NATO is undergoing a paradigm shift which is characterized by re-building defence capabilities and by keeping the door open for meaningful dialogue with Russia. The article describes the changes NATO went through since Spring 2014 and asks for the consequences the paradigm shift will have for Germany - which played a significant role in preparing the paradigm shift. Special emphasis will be laid on the commitments made in the field of military capabilities and defence investment. Also, Germany's role as a framework nation will be dealt

*Kontakt: Brigadegeneral a.D. Rainer Meyer zum Felde, Institut für Sicherheitspolitik an der Universität Kiel, email: meyerzumfelde@ispk.uni-kiel.de. with. The article concludes by pointing to mid-term consequences of a NATO that puts renewed emphasis on the deterrence and collective defence.

Keywords: NATO, defence strategy, deterrence, Germany

\section{Einleitung}

Nachdem die NATO für zwei Jahrzehnte primär eine politische Allianz war, die ihre Hauptaufgabe darin sah, international zur Krisenbewältigung beizutragen und ein Forum der Partnerschaft mit Russland, der Ukraine und anderen Ländern zu sein, vollzieht sich seit März 2014 ein Paradigmenwechsel. Auslöser war die Besetzung und Annexion der Krim durch Russland sowie der Versuch von russischen Milizen und Spezialtruppen, Teile der Ukraine im Wege einer hybriden Aggression der Kontrolle der Regierung in Kiew zu entringen. Die vorliegende Analyse zeichnet den Weg der NATO seither nach und befasst sich mit der Frage, in welcher Hinsicht die Notwendigkeit besteht, wieder eine Abschreckungs- und Verteidigungsfähigkeit der NATO herzustellen und was diese Entwicklung für Deutschland bedeutet. ${ }^{1}$

Im Einzelnen wird wie folgt vorgegangen: Nach einer Analyse der Bedrohungslage, insbesondere mit Blick auf Russland, werden die Anpassungen der NATO an die neue Lage nach 2014 dargestellt und analysiert, wobei die Unterschiede zu der vergangenen Periode herausgestellt werden, in der die Partnerschaft mit Russland als primäres

1 Der Verfasser war von Juli 2013 bis September 2017 als Angehöriger der Deutschen NATO-Vertretung tätig und hat die deutschen Interessen nach Weisung der Bundesregierung im Ausschuss für Verteidigungspolitik und -Planung (Defence Policy and Planning Committee, DPPC) vertreten. Nicht zuletzt in diesem Ausschuss wurden militärische Überlegungen, Schlussfolgerungen und Empfehlungen der NATO-Militärbehörden aus politisch-militärischer Sicht bewertet und damit die politischen Entscheidungen des Nordatlantikrats zur Anpassung des Verteidigungsdispositivs der Allianz sowie deren Indossierungen durch die Staats- und Regierungschefs bei den Gipfeltreffen von Wales im September 2014 und Warschau im Juli 2016 vorbereitet. 
Ziel galt und die Kernfunktion „Kollektive Bündnisverteidigung “ in den Hintergrund trat. In einem weiteren Schritt wird danach gefragt, was der Paradigmenwechsel der NATO - an dessen Zustandekommen die Bundesregierung maßgebend beteiligt war - für die deutsche Sicherheitspolitik und die Bundeswehr bedeutet. Dabei wird vor allem auf die Zusagen Deutschlands eingegangen, ambitionierte NATO-Fähigkeitenziele zu erfüllen und das Verteidigungsinvestitionsversprechen von Wales zu einzuhalten. Außerdem wird Deutschlands Rolle als Rahmennation behandelt. Abschließend werden weitergehende Perspektiven einer wieder stärker an Abschreckung und Bündnisverteidigung orientierten NATO aufgezeigt.

\section{Worin liegt die (potentielle) Bedrohung Europas durch Russland?}

Seit 2014 ist Russland offen zur Abkehr von der strategischpartnerschaftlichen Kooperation und zur hybriden Konfliktaustragung gegenüber dem Westen übergegangen. Anzeichen hierfür gab es schon früher: hierzu gehörte Präsident Putins Rede bei der Münchner Sicherheitskonferenz im Jahr 2007 und ein Jahr später die militärische Intervention in Georgien. Diese Ereignisse wurden in den meisten westlichen Hauptstädten jedoch nur unzureichend wahrgenommen oder fehlinterpretiert und erst im Nachhinein als Vorboten eines Gezeitenwechsels verstanden. Russland wird seit seinem aggressiven und völkerrechtswidrigen Vorgehen gegenüber der Ukraine im Frühjahr 2014 von nahezu allen westlichen Hauptstädten als zumindest potentielle Bedrohung für die staatliche und territoriale Integrität seiner Nachbarn angesehen. Der Kreml ist vom vormals strategischen Partner zum potentiellen Gegner in einem regionalen Konflikt in Nordosteuropa und/oder Südosteuropa geworden. ${ }^{2}$ Dieser Politikwechsel Russlands macht es für die NATO erforderlich, ihre Fähigkeit zur kollektiven Bündnisverteidigung wiederherzustellen.

Russland hat im Konflikt mit der Ukraine erkennbar werden lassen, wie weitgehend die seit etwa 10 Jahren laufenden Modernisierungsmaßnahmen den russischen Streitkräften neue Fähigkeiten verliehen haben. Es handelt sich nicht mehr um die trägen, auf Wehrpflicht basierenden, schlecht ausgerüsteten und wenig geübten Streitkräfte, deren mangelnde Leistungsfähigkeit noch im Georgienkonflikt 2008 offenkundig waren. Vielmehr war

2 Vgl. Adomeit 2017. das russische Militär im Sommer 2014 in der Lage, nach kurzer Vorbereitung der politischen Führung ein breites Spektrum militärischer Optionen anzubieten und über Monate hinweg aufrechtzuerhalten: von der (tatsächlich erfolgten) raschen Wegnahme der Krim über eine (tatsächlich erfolgte) hybride Operation gegen den Osten der Ukraine - bestehend aus begrenzter verdeckter Destabilisierung des Donbass - bis hin zu klassischen Operationen als Drohkulisse aufmarschierter regulärer Großverbände. Sie eröffneten die Option eines begrenzten Feldzugs entlang der Schwarzmeerküste bis Odessa oder Transnistrien und sogar zu einer Art „Blitzkrieg“ zur Besetzung Kiews. Russland hat damit seine Fähigkeit offenbart, regionale Nachbarstaaten mit hybrider Kriegführung - d.h. mit einer Kombination sowohl unkonventionell-verdeckter, als auch klassischer militärischer Operationen - ernsthaft zu bedrohen. Dieses Bedrohungspotenzial gefährdet nicht nur die territoriale Integrität anderer Staaten, es lässt sie auch politisch erpressbar werden. Dahinter steht eine ordnungspolitische Vorstellung, der zufolge Große Mächte das Recht haben, in ihrer Umgebung Interessenund Einflusszonen gegenüber kleinen Nachbarstaaten durchzusetzen. ${ }^{3}$

Jene kleineren NATO- und EU-Mitgliedstaaten, die an Russland angrenzen, hatten diese Befürchtung schon seit längerem artikuliert, damit aber wenig Gehör gefunden. Für sie bedeutete das Vorgehen Russlands gegen die Ukraine den offensichtlichen Nachweis der Berechtigung ihrer Befürchtungen. Entsprechend intensiv drangen sie ab März 2014 auf Unterstützung (auch militärisch) für die Ukraine. Vor allem aber forderten sie sofortige Schutzmaßnahmen für sich selbst, denn die Allianz habe es nun nachweislich mit einem anderen Russland zu tun, als bisher angenommen: Putins Russland sei bereit, bei sich bietender Gelegenheit ungewohnt hohe Risiken einzugehen und mit militärischer Gewalt Fakten zu schaffen. Es könne nach kurzer oder sehr kurzer Vorbereitung überraschend angreifen und es habe sich mit den seit Jahren laufenden Modernisierungen ein flexibles, schlagkräftiges Streitkräftedispositiv zugelegt, welches auf einen hybrid beginnenden Konflikt ausgerichtet sei, der aber möglicherweise rasch in konventionelle und hochintensiv geführte Operationen eskalieren könne, die von moderner Cyber- und Informationskriegführung begleitet wären.

3 Das russische Verständnis ähnelt der Breschnew-Doktrin der eingeschränkten Souveränität sozialistischer Länder, welches 1968 aus Anlass der Besetzung der Tschechoslowakei verkündet worden war. Es finden sich allerding auch Ähnlichkeiten mit Konzepten, die Carl Schmitt in den 40er Jahren des 20. Jahrhunderts entwickelt hatte, vgl. Krause 2017, 6. 
Die eigene militärische Lage wurde von diesen Ländern als prekär wahrgenommen: Keiner der exponierten kleineren Mitgliedstaaten in Nordost- und Südosteuropa verfügt über nationale Streitkräfte zur Landesverteidigung im Rahmen zivil-militärischer Gesamtverteidigungsplanung, welche (nach dem Vorbild Finnlands) gegenüber Russland einen gewissen eigenständigen Abschreckungseffekt hätten erzielen könnten. Dieses Defizit darf man ihnen nicht zum Vorwurf machen, denn diesen Verbündeten war schon während der NATO-Beitrittsprozesse im Zuge des Membership Action Plans stets angeraten worden, ihre begrenzten Ressourcen auf Beiträge zu fokussieren, die für Einsätze zum Konfliktmanagement oder für Stabilisierungsoperation geeignet seien (Balkan, Afghanistan, Nordafrika). Zudem gab es - abgesehen von der „generisch“ ausgerichteten, gerade einmal brigadegroßen damaligen NATO Response Force - keine Pläne oder gar existierende alliierte Kräfteformationen oder Verstärkungs-Großverbände, mit denen diese Verbündeten militärisch gegenüber einer russischen Aggression hätten geschützt werden können.

Zwar schlossen sich nicht alle Mitgliedstaaten der Bedrohungsperzeption der exponierten osteuropäischen Staaten in vollem Umfang an, aber innerhalb der Allianz gewann die Überzeugung Raum, dass es politisch notwendig sei, Moskau klar zu signalisieren, dass sich sein vermeintlicher Erfolg im Russland-Ukraine Konflikt gegenüber den durch Art. 5 geschützten Mitgliedstaaten nicht würde wiederholen lassen und dass die Allianz entschlossen sei, jeden etwaigen Test ihres Zusammenhalts und ihrer Handlungsfähigkeit in der Bündnisverteidigung $\mathrm{zu}$ bestehen - mit unabsehbaren Risiken für Russland für den Fall, dass es einen solchen Test wagen würde. ${ }^{4}$

Gleich wie man im Einzelnen die militärische Bedrohung interpretiert, die Russland darstellt, die strategische Lage in Europa hat sich nach Ansicht aller Mitgliedstaaten grundlegend verändert und dies hat Konsequenzen für alle Verbündeten. Wenn es um den Schutz der exponierten Verbündeten vor Russland geht, sind zwei Artikel des Nordatlantikvertrages von Bedeutung: Art. 3, der bestimmt, dass alle Bündnismitglieder die Verpflichtung haben, die für kollektive Bündnisverteidigung angemessenen Kräfte und Fähigkeiten zum eigenen und zum Schutz von Verbündeten bereitzuhalten; ${ }^{5}$ und Art. 5, der

\footnotetext{
4 Vgl. hierzu Gipfelerklärung von Wales Para 1-3.

5 Art 3 Nordatlantikvertrag: „Um die Ziele dieses Vertrags besser zu verwirklichen, werden die Parteien einzeln und gemeinsam durch ständige und wirksame Selbsthilfe und gegenseitige Unterstützung die eigene und die gemeinsame Widerstandskraft gegen bewaffnete Angriffe erhalten und fortentwickeln.“
}

bestimmt, dass im Falle eines militärischen Angriffs gegen einen Verbündeten dies als Angriff gegen alle Verbündeten angesehen und ihm mit bündnisgemeinsamer Verteidigung (collective defence) begegnet werden soll. ${ }^{6}$

Für Viele klingt das wie eine Neuauflage des Kalten Krieges und gerade in der deutschen politischen Debatte wird immer wieder gefordert, alles zu unternehmen, um einen neuen Kalten Krieg zu verhindern. Diese Sorge ist verständlich, doch lässt eine differenzierende Analyse erkennen, dass nur teilweise Übereinstimmungen mit der damaligen Lage bestehen:

a. Im Unterschied zum Kalten Krieg ist das Bedrohungspotential Russlands zwar vielfältiger und reicht von hybrider über konventionelle bis zu nuklearer Kriegführungsfähigkeit. Ein fundamentaler Unterschied besteht aber in seiner militärischen Reichweite: Russland ist nicht mehr, wie einst die Sowjetunion und der von ihr geführte Warschauer Pakt, zu einer großangelegten, raumgreifenden Aggression gegenüber (West-) Europa vom Nordkap bis zur Türkei in der Lage. Von den russischen Streitkräften, ihren operativen Planungen und militärischen Kräften und Fähigkeiten geht daher keine Bedrohung für die territoriale Integrität ganz Westeuropas bis hin zur Atlantik aus, wie dies in der zweiten Hälfte des 20. Jahrhunderts der Fall war. Die potentielle Bedrohung, soweit sie sich gegen die territoriale Integrität von Mitgliedstaaten richtet, ist regionaler Art, nämlich in Nordost- und/oder in Südosteuropa (allerdings mit Implikationen auch für rückwärtige Räume und vitale Seeverbindungen über Nordatlantik, Ostsee und Mittelmeer). Es bedarf daher auch keines militärischen Gegenkonzeptes der Allianz, wie dies im Kalten Krieg mit der Vorneverteidigung, einem statischen Verteidigungsdispositiv mit bereits im Frieden aneinandergereihten alliierten Großverbänden und einem bündnisweit bis in alle Einzelheiten durchgeplanten General Defence Plan der Fall war. Vielmehr gilt es, in einer möglicherweise militärisch hochintensiv ausgetragenen, aber auf eine

6 Art 5 Nordatlantikvertrag: „Die Parteien vereinbaren, dass ein bewaffneter Angriff gegen eine oder mehrere von ihnen in Europa oder Nordamerika als ein Angriff gegen sie alle angesehen wird; sie vereinbaren daher, dass im Falle eines solchen bewaffneten Angriffs jede von ihnen in Ausübung des in Artikel 51 der Satzung der Vereinten Nationen anerkannten Rechts der individuellen oder kollektiven Selbstverteidigung der Partei oder den Parteien, die angegriffen werden, Beistand leistet, indem jede von ihnen unverzüglich für sich und im Zusammenwirken mit anderen Parteien die Maßnahmen, einschließlich der Anwendung von Waffengewalt, trifft, die sie für erforderlich erachtet, um die Sicherheit des nordatlantischen Gebierts wiederherzustellen und zu erhalten“..... 
oder wenige europäischen Regionen begrenzten Auseinandersetzung NATO-seitig die Oberhand zu behalten. Nur so kann Russland von regionalen Aggressionen abgeschreckt werden. Es kommt darauf an, dass im gegnerische Risikokalkül die Einsicht obsiegt, einen solchen Konflikt nicht gewinnen zu können, und dass jeder Versuch, eine sich vermeintlich bietende Gelegenheit zu nutzen, mit unkalkulierbaren, jedenfalls $\mathrm{zu}$ hohen Risiken verbunden sein würde und daher nicht unternommen werden sollte. ${ }^{7}$

b. Aber auch für eine lediglich regionale Bedrohung gilt, dass manche Bestandteile des damaligen NATO-Verteidigungsdispositivs nach wie vor benötigt werden: Pläne, Kräfte und Mittel zur verzugslosen Reaktion auf ein begrenztes Austesten seitens Russlands sowie zur Führung von Anfangsoperation in einem ,contested environment", wenn dieses zur Abwehr eines begrenzten militärischen Angriffs notwendig wird. Dazu gehört neben nationalen Heimatschutzkräften ${ }^{8}$ des betroffenen Verbündeten und unverzüglich verlegten NATO-Reaktionskräften ${ }^{9}$ auch die schnellstmögliche Zuführung durchhaltefähiger Verstärkungskräfte zur Führung hochintensiver verbundener Land-, Luft- und Seeoperationen zur territorialen Verteidigung und Wiederherstellung des status quo ante. Das erfordert u. a. in Friedenszeiten Regionen-bezogene Planungen für die Gesamtverteidigung, sowie deren Einübung durch zivil-militärische Verfahrensübungen und die logistisch-infrastrukturelle Vorbereitungen für Truppenverlegungen.

c. Soweit sich die neue russische Bedrohung gegen die staatliche Integrität von Mitgliedstaaten und die funktionale Integrität westlicher Schlüsselinstitutionen wie NATO und EU mittels verdeckter Aktivitäten und Cyber-Konfliktaustragung richtet, hat sie hingegen eine neue, mit dem Kalten Krieg nicht vergleichbare Dimension und Reichweite. Diese Bedrohung ist offensichtlich nicht auf kleinere Verbündete in der Nachbarschaft Russlands beschränkt, sondern richtet sich auch auf Washington, London, Berlin, Paris und andere Hauptstädte mit dem Ziel, bei jeder sich bietenden Gelegenheit den Zusammenhalt der westlichen Verbündeten und den Fortbestand der regelbasierten westlichen Ordnung zu untergraben.

7 Vgl. Menkiszak 2017; Kamp 2017a.

8 National Home Defence Forces.

9 NATO Response Forces, NRF (mit der Very High Readiness Task Force, VJTF, als „Speerspitze”).
Aus dem vornehmlich regionalen Charakter der heutigen potentiellen Bedrohung von Mitgliedstaaten der NATO und der EU ergeben sich somit in vielerlei Hinsicht einerseits gänzlich andere Forderungen an die nationalen Verteidigungsdispositive der Mitgliedstaaten. Andererseits gelten wesentliche Charakteristik des Kalten Kriegs erneut: vor allem die Möglichkeit klassischer hochintensiver, raumgreifender Operationen mit schweren (gepanzerten) Großverbänden und die nicht auszuschließende Möglichkeit frühzeitiger nuklearer Eskalation. ${ }^{10}$

Ein hochintensiver militärischer Großkonflikt ${ }^{11}$ mit Russland, auch wenn er nur in regionaler Reichweite vorstellbar ist, wäre für die heutige NATO eine völlig neue Herausforderung, denn in den vergangenen zwei Jahrzehnten hat man sich auf ganz andere Szenarien eingestellt. ${ }^{12}$ Ein solcher Konflikt hätte vermutlich viele Ähnlichkeiten mit den militärischen Kampfhandlungen in den Weltkriegen des 20. Jahrhunderts; er würde sich jedenfalls fundamental und in nahezu jeder Hinsicht wesentlich von den Einsätzen niedriger und mittlerer Intensität ${ }^{13}$ zur Krisenvorbeugung und -Bewältigung unterscheiden, in denen die Streitkräfte der Allianz in den letzten 20 Jahren ihre Einsatzerfahrung gewonnen und worauf sie sich seither ausgerichtet haben. Vor allem in einem zentralen Kernelement ist die veränderte Lage gegenüber Russland mit der des Kalten Krieges gegenüber der Sowjetunion durchaus vergleichbar: Es geht um das Risikokalkül einer geopolitischen denkenden Großmacht, die ihre imperiale Stellung in Europa soweit wie möglich - auch unter Nutzung militärischer Macht gegenüber ihren Nachbarn - wieder festigen will und sich in dieser Hinsicht als Erbe der Sowjetunion sieht.

Die in der NATO mit den Gipfelbeschlüssen von Wales und Warschau erfolgte Renaissance des Doppelansatzes in der Harmel-Logik von „Wiederherstellung gestärkter Abschreckungs- und Verteidigungsfähigkeit“ und dem gleichzeitigen Bemühen um Wiederaufnahme eines „meaningfull dialogue“ mit Moskau ist daher kein Rückgriff in die Mottenkiste vergangener Epochen, sondern wohl durchdachtes Ergebnis einer nüchternen, differenzierenden Analyse der Ähnlichkeiten und Unterschiede zwischen damals und heute.

Während die Notwendigkeit, in Europa Krieg und militärische Konflikte durch Abschreckung und Verteidigungsfähigkeit $\mathrm{zu}$ unterbinden, das maßgebliche gemeinsame Merkmal von einst und jetzt ist, besteht ein

10 Vgl. Kamp 2017b.

11 In verteidigungsplanerischer Terminologie „Major Joint Operation Plus“ (MJO+).

12 Welche, wird nachfolgend unter Kapitel 3.1 dargestellt. 13 Smaller Joint Operations (SJO), Major Joint Operations (MJO). 
wesentlicher Unterschied zur Lage in den 70er und 80er Jahren des 20. Jahrhunderts darin, dass Europa zugleich auch anderen Herausforderungen begegnen muss. Auch wenn der Paradigmenwechsel der Allianz seit 2014 vor allem als Reaktion auf das Verhalten Russlands zu sehen sind, so hat die NATO in ihren Beschlüssen doch auch deutlich gemacht, dass sie sich der Vielfältigkeit der Herausforderungen bewusst ist. Ausdruck dieser Haltung ist, dass 2014 die zunächst drängendste Herausforderung - der potentiellen Bedrohung durch Russland mit Abschreckung, Verteidigung und zugleich Dialog entgegenzuwirken - in einen „360-Grad-Ansatz“ eingebettet wurde. Hierzu gehören die realen und akuten Herausforderungen Europas an der südlichen Peripherie mit allen ihren negativen Begleiterscheinungen und Auswirkungen (wie der Syrien-Krieg, islamistischer Terrorismus, die iranisch-saudi-arabische Rivalität, der Dschihadismus in der Sahelzone und in Ostafrika). Darüber hinaus kommt die Allianz in jüngster Zeit auch nicht umhin, die Proliferation von Kernwaffen (Nordkorea, Iran) sowie die zunehmende, auch militärische, Präsenz Chinas in Europas Nachbarschaft und in Europa selbst zur Kenntnis zu nehmen und sich damit auseinanderzusetzen. ${ }^{14}$

Zugleich steht alles Handeln der Europäer unter dem Vorbehalt des gleichbleibenden Interesses der USA am Fortbestand des transatlantic bond und der engen Zusammenarbeit mit den europäischen Verbündeten. Es gibt seit dem Amtsantritt von Donald Trump als 45. Präsident der USA immer deutlichere Anzeichen für ein zunehmendes Auseinanderdriften in Grundsatzfragen der globalisierten Welt. Hierzu gehören die immer schärfer gewordenen Differenzen über die verteidigungspolitische Lastenteilung ebenso wie über Handels- und Wirtschaftspolitik. Die Gefahr besteht, dass es auf dem Feld der Sicherheitspolitik zu einer Zersplitterung innerhalb der atlantischen Gemeinschaft kommt, die für den Zusammenhalt - dem „centre of gravity“ der atlantischen Allianz - höchst schädlich und gefährlich werden kann.

\section{Der Wandel der NATO seit 2014}

Das Erfolgsrezept der NATO im Kalten Krieg lautete: Sicherheit ist gleich Verteidigungsfähigkeit plus Entspannungsbemühungen $(\mathrm{S}=\mathrm{V}+\mathrm{E})$. Dahinter stand die Erkenntnis, dass sich Abschreckung nicht durch „Bluff“ vortäuschen lässt. Sie wirkt nur dann auf das Risikokalkül eines rational handelnden Gegners ein, wenn dieser

14 Vgl. Bruns/Kirchberger 2017. im Ergebnis aller seiner Informationen davon ausgehen muss, dass sein Gegenüber tatsächlich verteidigungswillig und verteidigungsfähig ist, und zwar in allen möglichen Konfliktformen von subversiv-verdeckter Art über die hybride und konventionelle bis hin zur nuklearen Ebene. In der Einschätzung eines Gegners müssen dazu neben dem politischen Willen zur Selbstbehauptung die notwendigen Bestandteile tatsächlich gebrauchsbereit vorhanden und sichtbar sein: Kräfte und Fähigkeiten (d.h. hinreichend umfangreiche und kampfkräftige Streitkräfte mit Verbänden und Großverbänden in geeigneten Strukturen mit entsprechender Ausbildung und multinationaler Interoperabilität), Mittel (d.h. Rüstung, Bewaffnung und Ausstattung mit modernem Material und möglichst überlegener Technik), Ausbildung und Übungen (Verfahrensübungen für Stäbe, zivil-militärische Gesamtverteidigungsübungen und Volltruppenübungen, aus denen sich letztlich überlegene Führungskunst der Stäbe und überlegenes militärisches Können der Truppe ergeben).

Im Kalten Krieg waren die Verteidigungsaufwendungen Deutschlands verglichen mit den heutigen gigantisch: Deutschland stellte mit 12 (statt heute 2) voll einsatzbereiten Heeresdivisionen und 36 (heute 6) Kampfbrigaden sowie entsprechenden Luft- und Seestreitkräften etwa die Hälfte der konventionellen Kampfkraft der NATO in Europa und wandte dafür in den 60er Jahren über $5 \%$, in den 70er Jahren über $4 \%$, in den 80er Jahren über 3\% seines BIP auf. ${ }^{15}$ Erst in den 90er Jahren sank der Verteidigungshaushalt zwecks „Friedensdividende“ auf unter 2\% ab. Dieser nachhaltig hohe Aufwand hat damals zum Erfolg geführt. Die Erfahrungen mit der Sowjetunion haben gezeigt, dass erfolgreiche Entspannungsbemühungen (KSE, Wiener Dokument, INF-Vertrag) und „meaningful dialogue“ dann erfolgreich sind, wenn für beide Seiten offensichtlich ist, dass ein „noch mehr“ an militärischer Stärke trotz ruinösen Aufwands keinen politischen Vorteil einbringt.

\subsection{Der Paradigmenwechsel der NATO in den neunziger Jahren}

Während der neunziger Jahre brach sich in der Allianz angesichts der grundlegend anderen strategischen Ausgangslage ein politischer, militärischer und struktureller Paradigmenwechsel Bahn, der mit dem „Signal von

15 Vgl. hierzu das vom damaligen Verteidigungsminister Helmut Schmidt herausgegebene Weißbuch 1971/1972, Tabelle S. 169 „Verteidigungsausgaben der NATO-Länder“: Deutschland 4,9\%(1961), 6,0 \% (1962), 6,5\% (1963), 5,9\% (1964), 5,5\% (1965), 5,3\% (1966), 5,6\% (1967), 4,7‘\% (1968), 4,7\% (1969), 4,3\% (1970), 4,4\% (1971). 
London“ 1991 begann und Ende des Jahrzehnts mit dem Strategischen Konzept 1999 einen vorläufigen Abschluss fand. ${ }^{16}$ Die NATO wandelte sich in zwei Jahrzehnten von einem System der kollektiven Bündnisverteidigung zu einer Sicherheitsorganisation, die ab Mitte der 1990er Jahre ihre Haupttätigkeit in der Krisenprävention, dem Krisenmanagement durch Stabilisierung „out of area“ und der kooperativen Sicherheit durch „Stabilitätstransfer nach Osten“ im Rahmen von Partnerschaften sah. Militärisch und militär-politisch drückte sich dies in den zivilmilitärischen Missionen IFOR, ${ }^{17} \mathrm{SFOR}^{18}$ und $\mathrm{KFOR}^{19}$ zur Stabilisierung Bosnien und Herzegowinas und des Kosovo sowie ab 2003 der ISAF ${ }^{20}$ Mission in Afghanistan aus. Die maßgeblichen strategischen Dokumente wurden beginnend mit der Gipfelerklärung von London 1990 („Hand zur Freundschaft“), der NATO-Russland Grundakte (1997) und dem Strategischen Konzept von 2010 geschaffen. Im nationalen Bereich zeigte sich dieser Wandel in Deutschland im Weißbuch 2006 und den Verteidigungspolitischen Richtlinien von 2011. Die Verteidigungshaushalte der Mitgliedstaaten fielen in dieser Zeit kontinuierlich unter die informelle Richtlinie von $2 \%$ des Bruttosozialproduktes ab und lagen 2013 bei fast allen Verbündeten unter 2\% (außer den USA, Großbritannien, der Türkei und Griechenland). Politisch und militärisch lag der „mindset“ der Allianz fast ausschließlich auf den wahrscheinlichsten Einsätzen und Missionen, nämlich Krisenmanagement im Stile von IFOR, SFOR, KFOR, ISAF in der südlichen Peripherie der Allianz mit dem Ziel der Bewahrung und Wiederherstellung regionaler Stabilität im Falle von failing states, sowie ab 2001 mit dem Ziel, in Afghanistan keinen sicheren Hafen für Terrorismus der al-Qaida-Ausprägung mehr zuzulassen.

Politisch bestand dieser erste Paradigmenwechsel der Allianz aus den folgenden Kernelementen:

1. Russland wurde nicht mehr als militärische Bedrohung und Gegner gesehen, sondern als strategischer Partner, dem die Hand zur Freundschaft geboten wurde. Damit einher ging eine „Politik der offenen Tür“ (Open Door Policy) gegenüber den ehemaligen Warschauer Pakt-Verbündeten der aufgelösten Sowjetunion, die wiederum mit einer Begleitstrategie für Russland verbunden war (in Form einer Grundakte, dem NATO Russia Founding Act). Mit dieser Grundakte sollte ein besonderes Partnerschaftsforum zwischen

16 Siehe zu dieser Epoche Meier/Roßmanith/Schäfer 2003, 258-281, sowie: Meier/Hannemann/Meyer zum Felde 2012, 332-333.

17 Implementation Force für die Dayton Peace Accords zu Bosnien und Herzegowina, $1995 \mathrm{ff}$.

18 Stabilisation Force (Nachfolgemission zu IFOR).

19 Kosovo Stabilisation Force.

20 International Stabilisation and Assistance Force den Mitgliedstaaten der Allianz einerseits und Russland andererseits geschaffen werden, das im NATORussland-Rat seinen Ausdruck fand.

2. Zentrales Ziel war der Stabilitätstransfer nach Osten in Form der - von den USA und Deutschland gemeinsam initiierten und vom damaligen NATO-Generalsekretär vorangetriebenen „Partnerschaft für den Frieden“, die mit einem Euro-Atlantic Partnership Council (EAPC) zu 45 Nationen, bestehend anfänglich aus den damaligen noch 16 NATO-Verbündeten und den zumindest an regelmäßiger Konsultation oder Zusammenarbeit mit der Allianz interessierten osteuropäischen Partnernationen.

3. Bedeutsam war auch die Einrichtung einer weiteren „Distinctive Partnership“ mit der Ukraine, nicht zuletzt als Gegenleistung - zusammen mit zugesagten Garantien der erhaltenen Souveränität - für deren Bereitschaft, das von der Sowjetunion als Erbmasse zugefallene Nukleararsenal zu verschrotten und auf den nuklearen Status zu verzichten.

4. Zugleich ging die Allianz von Abschreckung mittels statischer, territorialen Bündnisverteidigung innerhalb des NATO-Territoriums zu - von den Vereinten Nationen nach Kapitel VII (enforce compliance) mandatierten - Interventionen und Krisenreaktions-Missionen in und an der südlichen Peripherie Europas über, auf zunehmend strategische Entfernungen.

Dieser verteidigungspolitischen Neuausrichtung der Allianz folgte die Anpassung des gesamten Verteidigungsdispositivs der Allianz und der - an den NATO-Vorgaben orientierten - nationalen Verteidigungsdispositive ihrer Mitgliedstaaten:

1. Der NATO-Verteidigungsplanungsprozess basierte fortan nicht mehr auf einem bedrohungsorientierten Ansatz, der die militärischen Kräfte und Fähigkeiten Russland im Blick hatte, sondern wandelte sich zu einem rein „Fähigkeiten-orientierten Ansatz“, der nur noch an jenen eigenen Kräften und Fähigkeiten ausgerichtet war, die der Allianz von den Mitgliedstaaten für planerische Zwecke auf Grundlage von möglichen Szenarien angezeigt wurden.

2. Ebenso wurden sämtliche Planungs- und Übungsszenarios hinsichtlich der „roten Übungspartei“ umgestellt auf fiktiv-generische Bezeichnungen, um zu gewährleisten, dass Russland nicht mehr als militärische Bedrohung angenommen wurde. Die letzte große Gesamtverteidigungs-Verfahrensübung im Rahmen der Allianz auf Basis einer angenommenen Bedrohung konkret durch Sowjetunion/Russland fand 1989 mit der Übung WINTEX/CIMEX statt. Seither hat es 
vergleichbare Übungen mit Russland als angenommenem Gegner nicht mehr gegeben. Dies ist deswegen von großer Bedeutung, weil mit diesem Wandel vom konkreten „Feindbild“ des Kalten Krieges zum „generischen“ Übungsverhalten auch das Bewusstsein für die Notwendigkeit dahinschwand, die Fähigkeit zur Bündnisverteidigung am hochintensiven Ende des Spektrums gegen einen nahezu gleichwertigen Gegner aufrecht zu erhalten. Erst 2013 wuchs im Rahmen der "Connected Forces Initiative“ die Erkenntnis, dass die Streitkräfte der Allianz im Kontext ihrer langjährigen Krisenmanagement-Einsätze IFOR, SFOR, KFOR und ISAF (und dabei der Fokussierung des Anspruchsniveaus auf maximal „Operationen zur Bekämpfung Aufständischer“ der Ebene Zug, Kompanie, Bataillon) sich in ihren Fähigkeiten verengt hatten und Ausbildungs-, Führungs- und Ausrüstungsmängel mit Blick auf Operationen der Ebene Brigade, Division und Korps erkennen ließen. In zwei Jahrzehnten hatten Führungsstäbe wie Truppe ihr vormals hohes handwerkliches Können mit Blick auf hochintensive militärische Operationen am obersten Ende des Spektrums weitgehend verloren; diese Fehlentwicklung bedurfte einer Korrektur.

3. Das militärische Anspruchsniveau (Level of Ambition) wurde auf die Fähigkeit zur Führung von 2 Major Joint Operations (MJO, jeweils maximal Korps-Größe) sowie von 6 Smaller Joint Operations (SJO, jeweils maximal Brigade/Bataillons-Größe) zurückgenommen. Inhaltlich trat die Bekämpfung bewaffneter Aufständischer (Counter Insurgency Operations, COIN) im Rahmen von Stabilisierungsmissionen als wahrscheinlichste und häufigste Aufgabe in den Vordergrund. Hierauf richteten sich beginnend ab Mitte der 90er Jahre zunehmend die nationalen Verteidigungsdispositive und damit faktisch auch das militärische Gesamtdispositiv der Allianz aus. Die Integrierte Kommandostruktur (NATO Command Structure, NCS) wurde 2003 und 2010 in zwei drastischen Schritten von ihrer vormaligen Kalte-Kriegs-orientierten Struktur zur Verteidigung NATO-Europas gegen eine großangelegte Aggression zugunsten einer funktionalen Struktur (Allied Command Operation ACO, Allied Command Transformations ACT) grundlegend umgestaltet. Diese wurde auf die Führung von komplexen zivil-militärischen Krisenmanagement Missionen nach dem comprehensive approach und entsprechend dem Level of Ambition ,2+6“ umstrukturiert und dabei zugleich auf etwa die Hälfte ihres früheren Personalumfangs reduziert. Auch wenn weiterhin das Bekenntnis zur Notwendigkeit einer Abschreckungs- und Verteidigungsfähigkeit aufrecht erhalten blieb, wurde dies in der Praxis nicht mehr unterfüttert - auch nicht durch Übungen von Truppen oder durch Verfahrensübungen.

Alle diese Entwicklungen reflektierten die Tatsache, dass Russland seit Mitte der 90er Jahre von der Allianz nicht nur deklaratorisch, sondern auch faktisch mit allen Konsequenzen als strategischer Partner, PfP-Partnernation und $\mathrm{NRC}^{21}$-Partner wahrgenommen wurde und nicht mehr als potentielle Bedrohung. ${ }^{22}$

Vor diesem Hintergrund bestand im Jahre 2013 die allgemeine Erwartung, dass mit dem absehbaren Ende der NATO-geführten Missionen auf dem Balkan (KFOR) und in Afghanistan (ISAF), der allgemeinen Einsatzmüdigkeit der Mitgliedstaten angesichts des enttäuschenden Aufwand/ Ergebnis Resultats der ISAF-Mission und der begrenzten Eignung militärischer Lösungen für die schwierigen Stabilisierungsprobleme in Nordafrika und dem weiteren mittleren Osten die Ära der expeditionary operations der Allianz zu einem Ende kommen werde, wie auch die frühere Primäraufgabe von Abschreckung und Verteidigung im Osten bereits Ende der 90er Jahre dauerhaft zum Abschluss gebracht worden war. Mit dieser vorherrschenden Einschätzung richtete sich Ende 2013 die NATO darauf ein, zu einer politischen Organisation mit Schwerpunkt im Bereich kooperativer Sicherung durch Partnerschaft, der dritten Säule des Strategischen Konzepts von $2010^{23} \mathrm{zu}$ werden. Auch sollte das lange schwelende Dauerthema fairer transatlantischer und innereuropäischer Lastenteilung unter den Verbündeten mit Blick auf Verteidigungshaushalte, Kräftebereitstellungen und Schließung erkannter Fähigkeitsdefizite bei Krisenmanagementmissionen einer Lösung zugeführt werden. Als drittes übrig bleibendes Hauptthema galt es unter der Überschrift Connected Forces Innitiative (CFI) ${ }^{24}$ aus grundsätzlichen Erwägungen - noch ohne dezidierte Bedrohungsanalyse und konkreten Bezug zu Russland -, die Streitkräfte der Verbündeten und die Stäbe der NATO neben den laufenden Einsätzen auch wieder in der Führung von höherintensiven militärischen Operationen auf den Ebenen oberhalb Bataillon - „Gefecht der verbundenen Waffen“

21 NATO Russia Council, NRC.

22 Zur NATO in der Zeit von 2006 bis 2012 siehe: Meier/Hannemann/ Meyer zum Felde 2012, 328-364.

23 Active Engagement, Modern Defence. Strategic Concept for the Defence and Security of the Members of the North Atlantic Treaty Organization. Adopted by Heads of State and Government at the NATO Summit in Lisbon, 19-20 November 2010. Siehe auch Gipfelerklärung von Lissabon, para 2.

24 Die Connected Forces Initiative („Streitkräfte im Verbund“) wurde beim NATO-Gipfel in Chicago 2012 beschlossen 
im teilstreitkraftübergreifenden Verbund - zu üben und das verlernte handwerkliche Können am oberen Ende des theoretischen Einsatzspektrums wieder aufzufrischen.

So wurde die Zukunft der Allianz im Zurückgehen auf einen Bereitschafts,- Trainings- und Übungsbetrieb einerseits und intensivierte politische Konsultationen zu einem breiten politischen Themenfeld andererseits gesehen. Nur von wenigen Nationen, u.a. Großbritannien, Polen und den baltischen Staaten, wurden bereits im Herbst 2013 die groß angelegten Übungsaktivitäten Russlands (ZAPAD 2013) als bedenklich thematisiert und die im Vergleich unterproportionalen NATO-Ausbildungs- und Übungsaktivitäten kritisch hinterfragt.

\subsection{Der neue Paradigmenwechsel der NATO}

Das aggressive Vorgehen Russlands gegen die Ukraine ab März 2014, die völkerrechtswidrige Besetzung und Annexion der Krim und damit die Veränderung territorialer Grenzen in Europa mit militärischer Gewalt erstmals seit Ende der 40er Jahre sowie die anhaltende Destabilisierung des Ostteils der Ukraine mit Mitteln hybrider Kriegführung bei gleichzeitigem Aufmarsch von russischen Großverbänden traf die Allianz unvorbereitet. Die Aktionen Russlands waren für die NATO und ihre Mitgliedstaaten eine strategische Überraschung. Mit dem russischen Vorgehen wurden alle Abmachungen und Vereinbarungen, auf denen die internationale Friedensordnung nach Ende des Kalten Krieges beruhte, von Russland verletzt und in Frage gestellt - mit dem Ergebnis eines tiefgreifenden und nachhaltigen Vertrauensverlustes in Russland als berechenbaren Partner. ${ }^{25}$ Die gegenüber Russland exponierten Verbündeten forderten mit Nachdruck sofortige Reaktionen der NATO sowohl zum Schutz des Nichtmitglieds Ukraine, vor allem aber für sich selbst. Russland wurde von da an zumindest von den osteuropäischen Nationen als eine reale Bedrohung ihrer eigenen Sicherheit gesehen und von allen übrigen Verbündeten zumindest als eine „potentielle“ Bedrohung bewertet.

Zeitgleich kam es im Irak und Syrien zum Auftreten des Islamischen Staates (ISIL/DAESH). Damit trat eine neue Kategorie von Akteur auf, „non-state actor with state-like aspirations", und vor allem die südlichen Mitgliedstaaten der NATO sahen sich mit ebenfalls hybriden, dennoch gänzlich anders gearteten Bedrohungen konfrontiert, bzw. mit deren Folgen: Flüchtlingsströme und ungesteuerte Migration über die Balkanroute sowie das Mittelmeer nach Europa. Dies waren für die betroffenen

25 Vgl. Krause 2017, 9.
Staaten Südeuropas nicht nur potentielle, sondern sehr „reale“ Bedrohungen der Stabilität. ${ }^{26}$

Die Schrecksekunde über das strategische Überraschungsmoment durch Russland fiel für eine konsensbasierte Großorganisation, in der sich 28 Hauptstädte mit jeweils unterschiedlichen Interessenlagen und Perzeptionen auf ein gemeinsames Lagebild und gemeinsame Reaktionen verständigen mussten, verhältnismäßig kurz aus. Bereits nach wenigen Wochen herrschte Einvernehmen, dass es sich nicht um ein vorübergehendes Gewitter, sondern um einen Gezeitenwechsel handelte, der als Antwort der Allianz unausweichlich einen weitreichenden Paradigmenwechsel - zumindest in wesentlichen Teilbereichen - erforderlich werden ließ. Als unmittelbare Sofortreaktion mit Blick auf die Ukraine entschloss sich die Allianz einvernehmlich, nicht zuletzt auf starkes deutsches Einwirken hin, nicht die Rolle des "first responders“ einzunehmen, sondern dies der Europäischen Union mit deren Möglichkeiten zur Verhängung wirtschaftlicher Sanktionen zu überlassen. Die Allianz schloss sich vielmehr dem von der EU und anderen Organisationen gesetzten Ton beim Verdammen des russischen Verhaltens an, ohne darüber hinauszugehen. Die damit verbundene Absicht war, nicht zu einer möglichen Eskalation beizutragen, solange nicht die Sicherheit der von Artikel 5 Washingtoner Vertrag geschützten Mitgliedstaaten direkt bedroht erschien. Allerdings entschloss sich die Allianz auf Betreiben vor allem der osteuropäischen Verbündeten, als Zeichen der Solidarität mit der Ukraine die Arbeit des NATO-Russlandrats stark einzugrenzen und auf der militärischen Ebene vollständig zu suspendieren. Damit sollte signalisiert werden, dass es bis zur Wiederherstellung des Status quo ante gegenüber Russland keine Rückkehr zu „business as usual“ geben könne. Diesen weitreichenden Entschluss der faktischen Suspendierung des - eigentlich für gute wie für schlechte Zeiten gedachten - NATO-Russland-Rats als Dialogforum muss im Nachhinein als ebenso kurzfristige wie kurzsichtige Konsensentscheidung der NATO durch deren Außenminister unter den Extremumständen des Frühjahrs 2014 gewertet werden. Aus heutiger Sicht wäre es besser gewesen, die NRC-Arbeit inhaltlich einzugrenzen, aber nicht auf der militärischen Ebene gänzlich zu suspendieren.

Neben dieser - insbesondere von Deutschland aktiv betriebenen - insgesamt zurückhaltenden Reaktion der Allianz auf das aggressive Vorgehen Russlands gegenüber der Ukraine begann das Bündnis gleichwohl unverzüglich, in den Gremien der Allianz eine weitreichende Anpassung an die von Grund auf veränderte Lage vorzunehmen:

26 Vgl. Gipfelerklärung von Wales, para 32-39 
Im Gesandtenausschuss und im politischen Ausschuss wurden die politischen Implikationen im Umgang mit Russland sowie die notwendigen institutionellen Anpassungen beraten, im Ausschuss für Verteidigungspolitik und -Planung sowie im NATO Militärausschuss wurden auf Grundlage der Vorschläge des SACEUR Beschlussvorlagen zu den notwendigen militärischen Anpassungsmaßnahmen erarbeitet. Damit gewann der in 2013 zunächst als Routineveranstaltung entworfene, mit einer relativ leichten Agenda geplante Gipfel in Wales für September 2014 eine ungeahnte historische Bedeutung. Auf ihm mussten nun plötzlich die Weichen für einen zweiten, grundlegenden und langfristigen Paradigmenwechsel vor allem mit Blick auf Russland gestellt werden. Zweck war nun, die Handlungsfähigkeit der Allianz unter Beweis zu stellen, ohne sie im Russland-Ukraine-Konflikt militärisch aktiv werden zu lassen. Der Gipfel sollte mit einem Bündel von Sofortmaßnahmen für die Steigerung der Reaktionsfähigkeit der Allianz zum Schutz der Mitgliedstaaten (allerdings nicht zum Schutz von Partnernationen wie Ukraine und Georgien) sorgen und gleichzeitig die gebotene Rückversicherung (reassurcance) der verunsicherten osteuropäischen Verbündeten bewirken ${ }^{27}$.

Im Bereich der militärischen Anpassung standen hierbei im Wesentlichen zwei Optionen im Mittelpunkt der Diskussion:

- Den Russland gegenüber exponierten osteuropäischen Nationen schwebte anfänglich die Idee einer im Osten aufzubauenden Vorneverteidigung vor, nach dem Modell ständiger stehender Kräfte wie im Kalten Krieg entlang der innerdeutschen Grenze.

- Von Deutschland, den USA, Großbritannien und Frankreich wurde gemeinsam die Alternativversion vertreten und von zahlreichen südlichen Nationen unterstützt, auf Erhöhung der „responsiveness“ zu setzen, ohne mit großem Ressourceneinsatz substanzielle Kampfverbände permanent an den Ostgrenzen der NATO zu binden. Ausschlaggebend war für Deutschland das aus übergeordneten politischen Gründen gewollte Festhalten an den Bestimmungen der NATO/Russland-Grundakte als letztem verbliebenen Element des zusammengebrochenen Gebäudes internationaler Abmachungen mit Russland. Dieses politische Grundlagendokument schloss die permanente Stationierung substantieller Kampfverbände (in russischer Auslegung: eine alliierte Brigade pro Land) aus. Den USA, Großbritannien und Frankreich ging es vor allem darum, ihre Streitkräfte in Europa verlege- und einsatzbereit in alle Richtungen $\mathrm{zu}$

27 Vgl. hierzu Gipfelerklärung von Wales, para 16-23 halten und nicht statisch an eine Region zu binden. Zugleich wurde Einvernehmen hergestellt, dem starken Bedürfnis der osteuropäischen Verbündeten nach Rückversicherung für den Fall einer Bedrohung durch Russland zu entsprechen. An dieser Konstellation und den daraus folgenden politischen Vorgaben durch den Nordatlantikrat orientierte sich der NATO Oberbefehlshaber SACEUR mit seinen militärischen Überlegungen.

Unter Berücksichtigung der Vorgaben des Nordatlantikrates und im Bewusstsein der Diskussionen auf der politischen Ebene legte SACEUR im Sommer 2014 seine militärischen Vorschläge für „reassurance“ und „short- and mid-term adaptation measures" vor, die als militärischer Ratschlag der NATO Militärbehörden im Militärausschuss beschlossen und im Ausschuss für Verteidigungspolitik und Planung aus politisch-militärischer Sicht bewertet wurden. Das gesamte Maßnahmenpaket wurde anschließend vom Nordatlantikrat als Readiness Action Plan gebilligt und beim Gipfel in Wales im September 2017 als wichtigstes Beschlusspaket von den Staats- und Regierungschefs indossiert. ${ }^{28}$

Die Umsetzung der einzelnen Elemente erfolgte unverzüglich und in weniger als 24 Monaten. Im Wesentlichen waren dies: die Beschleunigung der politischen und militärischen Entscheidungsprozesse in der Allianz, die Erarbeitung von „graduated response plans“, die Aufwertung und der Ausbau des multi-nationalen Korpsstabs Nord-Ost als Zentrum für alle Angelegenheiten kollektiver Verteidigung in Nordosteuropa, der Aufbau von logistischen Aufnahmestäben (NATO Force Integration Units, NFIUs) in den osteuropäischen Staaten („NATO flag on the soil"), vermehrtes Air Policing durch die Verbündeten in den baltischen Staaten, verstärkte maritime Präsenz in der Ostsee, sowie eine höhere rotierende Übungspräsenz in den östlichen Mitgliedstaaten. Vor allem aber sollte als Zeichen deutlich erhöhter Responsiveness der Ausbau der NATO Response Force zu einer Divisions-großen Joint Task Force erfolgen, die eine multinationale, Brigade-große „Speerspitze“ umfasste: die Very High Readiness Joint Task Force (VJTF) mit einer nur wenige Tage Vorbereitung erfordernden Einsatzbereitschaft.

Neben dem Readiness Action Plan wurde in Wales das alte Anliegen einer ausgewogeneren transatlantischen und innereuropäischen Lastenteilung, nun mit höchster Priorität, erneut aufgenommen. Dabei gingen alle Teilnehmer davon aus, dass angesichts der akut veränderten Lage eine langfristige Trendumkehr bei den gesunkenen Vertei-

28 Gipfelerklärung von Wales, para 5-12 
digungsaufwendungen der Mitgliedstaaten einzuleiten sei. $\mathrm{Zu}$ diesem Zweck wurde der Defence Investment Pledge ${ }^{29}$ verabschiedet, ein Verteidigungsinvestitionsversprechen, welches den Verpflichtungen unter Art. 3 des Nordatlantikvertrags gerecht werden sollte. Dazu wurden auf höchster Ebene und unter voller Einbeziehung der Außen- und der Verteidigungsminister drei politische Schritte vereinbart, die alle mit dem Buchstaben „““ anfingen:

1. „Cash“ im Sinne von Steigerung der Verteidigungshaushalte auf $2 \%$ vom BIP und dabei $20 \%$ für Investitionen als Richtwert;

2. „Capabilities“ im Sinne der Erfüllung der den Nationen zugewiesenen NATO-Fähigkeitenziele des NATOVerteidigungsplanungsprozesses;

3. „Contributions“ im Sinne von fortgesetzten Kräfte- und Fähigkeitenbeiträgen zu Einsätzen bei den laufenden Stabilisierungsmissionen im Süden wie auch bei den neuaufgelegten Maßnahmen zur Rückversicherung im Osten.

Der Defence Investment Pledge wurde als Kompromiss zwischen denen, die mehr wollten, und denen, die sich zu weniger verpflichten wollten, von Deutschland vermittelt, mit mehrfachen „Caveats“, welche den Konsens aller 28 Regierungen ermöglichten. Dazu gehörten:

- Es handelte sich um ein Versprechen, aber keine bindende Verpflichtung („pledge“);

- Es sollte zu einer gleichwertigen Berücksichtigung sowohl von Input- (cash, capabilities) als auch OutputLeistungen der Nationen (commitments and contributions to missions) kommen;

- Die Richtzahlen von 2\% Verteidigungsausgaben vom BIP und 20\% Anteil der Investitionsausgaben am Verteidigungsetat sollten als Richtwerte (,guideline“) verstanden werden. Wichtig war, dass eine Trendwende und zumindest eine Annäherung (,aim at moving towards $2 \%$ ") an die Richtzahlen und erfolge. Es wurde ein Zeitrahmen von 10 Jahren veranschlagt, innerhalb dessen die Richtwerte zu erreichen seien („within a decade“). Allerdings sollten die Richtwerte nicht mechanistisch und als Selbstzweck verstanden werden, deshalb kam es zu einer Bindung an die Erfüllung der vereinbarten NATO-Fähigkeitenziele (,to meet NATO capability targets“).

Das Verteidigungsinvestitionsversprechen im von Deutschland eingebrachten Wortlaut wurde auch bei den folgenden Treffen der Staats- und Regierungschefs in Warschau (2016) und Brüssel (Mai 2017) in vollem Umfang

29 Gipfelerklärung von Wales, para 14-15 und ohne Abänderungen bekräftigt und seine Umsetzung vorangetrieben. Vor allem das Element „2\%“ blieb dabei allerdings politisch umstritten; während einige dieses Ziel als politisch von zentraler Bedeutung und unbedingt zu erfüllen ansehen, betrachten andere Verbündete eine lediglich tendenzielle Annäherung an die 2\% bereits als Erfüllung des Versprechens von Wales.

Das in Wales gebilligte Sofortmaßnahmenpaket mit der Zielsetzung erhöhter Responsiveness wurde bereits kurz nach Beginn seiner Implementierung mit einem weit in die Zukunft weisenden Planungsdokument komplementär ergänzt, der Political Guidance for Defence Planning. Sie enthielt als Kernaussage die politische Vorgabe, den Schwerpunkt der Planung wieder auf die Bündnisverteidigung und die dafür erforderlichen Kräfte und Fähigkeiten zu setzen (re-newed emphasis on collective defence), und wurde bereits im Juni 2015 nach intensiven Verhandlungen im DPPC vom Rat verabschiedet. Damit wurde die mittel- und langfristig wirkende NATO-Verteidigungsplanung umgesteuert von der Schwerpunktsetzung auf leichte verlegbare Kräfte zur Bewältigung des bis dahin gesetzten Level of Ambition (2 MJO, 6 SJO) hin zu der Fähigkeit, darüber hinaus auch wieder eine Major Joint Operation Plus ( $\mathrm{MJO}+$ ), in einem hoch-intensiven regionalen Großkonflikt mit einem modern ausgestatteten Gegner führen zu können.

Zugleich ergab sich daraus, nach dem ebenfalls in Wales beschlossenen Rahmennationenkonzept (Framework Nations Concept $)^{30}$, dass die um Deutschland als Rahmennation gebildete Gruppe von anfänglich 10 europäischen Nationen künftig nicht nur gemeinsam in Capability Clusters an der Schließung von Fähigkeitenlücken in 16 prioritären Feldern arbeiten, sondern darüber hinaus auch gemeinsam so schnell wie möglich die gemeinsame Bereitstellung von einsatzbereiten Großverbänden leisten sollte, welche der enhanced NRF-Division als nächste Verstärkungskräfte folgen könnten.

Neben diesen wesentlichen europäischen Aktivitäten entschlossen sich die USA mit ihrer European Reassurance Initiative (ERI), ihre bis dahin in Europa stehenden zwei Brigaden auf eine verstärkte Division mit insgesamt 4 Kampfbrigaden aufzustocken und über den Atlantik einzuführende Verstärkungskräfte einzuplanen. Dies war ein starkes politisches Signal der Zuwendung zu Europa nach vorangegangenen Jahren des Abzugs im Kontext des von Obama und Clinton verkündeten „Pivot to Asia“.

Im Lichte der Implementierung dieser Beschlüsse von Wales und den fortlaufenden Bewertungen in den militä-

30 Gipfelerklärung von Wales, para 67. Siehe hierzu auch Glatz/Zapfe 2017. 
rischen und politischen Gremien der Allianz zum Stand der Implementierung wurde im Laufe des Jahres 2015 der ursprünglich hauptsächlich gegen Russland ausgerichtete Ansatz des RAP zunehmend im sog. $360^{\circ}$-Ansatz auf alle strategischen Richtungen, insbesondere auch den Süden, ausgeweitet mit dem Ziel, den dortigen, sehr unterschiedlichen Herausforderungen durch den islamistischen Terrorismus und das Auftreten von non-State Actors with State-like Aspirations wie ISIL/DAESH ebenfalls begegnen zu können.

Mit Blick auf Russland stellte sich, auch im Kontext einer Szenario-basierten Verfahrensübung, zu Beginn des Jahres 2016 heraus, das der RAP aufgrund bestimmter russischer Fähigkeiten (anti-access/area denial) in dem Kaliningrad Oblast mit Blick auf die Ostsee und in der rasch militarisierten Krim mit Blick auf das Schwarze Meer auf Dauer nicht ausreichte. In Vorbereitung des Gipfels von Warschau im Juli 2016 wurden daher zwei weitere, intensiv verhandelte strategische Grundlagendokumente der zweiten Ebene (bei anhaltender Gültigkeit des Strategischen Konzepts von 2010) erarbeitet. Mit ihnen sollten im ersten Schritt die Prinzipien und Charakteristika glaubwürdiger Abschreckung und Verteidigung - unter Berücksichtigung der Erfahrungen aus dem Kalten Krieg, aber übertragen auf die anders gearteten Bedingungen des 21. Jahrhunderts - eingehend analysiert und, darauf aufbauend im zweiten Schritt, ein angemessen gestärktes Abschreckungs- und Verteidigungsdispositiv der Allianz entworfen wurde. Mit diesen Grundlagendokumenten wurde ein weiteres, über den RAP deutlich hinaus gehendes Maßnahmenpaket beschlossen und in Warschau von den Staats- und Regierungschefs gebilligt. Dieses bestand aus den folgenden Elementen:

- Einrichtung von vier Gefechtsverbänden (combat battle groups) nach dem Vorbild der alliierten Präsenz in Westberlin in den drei baltischen Staaten und Polen als enhanced Forward Presence ${ }^{31}$;

- Einrichtung einer maßgeschneiderten Präsenz (tailored Forward Presence) für Rumänien, Bulgarien und der Türkei mit Blick auf den Schwarzmeerraum;

- dazu Erarbeitung einer Verstärkungsstrategie mit follow-on forces als Verstärkungskräften über die enhanced NRF hinaus.

Ebenso wie der RAP nach Wales, wurde auch dieses Beschlusspaket mit Hochdruck implementiert und die

31 Jeweils in der Größenordnung eines verstärkten Bataillons, somit unterhalb der Schwelle substanzieller Kampfverbände nach russischer Auslegung des NATO-Russia Founding Act und damit weiterhin in dessen Rahmen.
Umsetzungsmaßnahmen mit Blick auf ihren Fortschritt mit großer Aufmerksamkeit durch Berichte an die Verteidigungsminister in allen nachfolgenden VM-Treffen gesteuert.

Parallel dazu gelang es, im Rahmen des ebenfalls angepassten und modernisierten NATO-Verteidigungsplanungsprozesses, die Nationen zur Annahme ambitionierter NATO-Fähigkeitenpakete zu bewegen, mit denen eine mittel- und langfristige Wiederherstellung der Abschreckungs- und Verteidigungsfähigkeit auch am hochintensiven Ende des Spektrums gewährleistet werden kann.

In diesem Zusammenhang wurde ferner die bereits in Wales vereinbarte Verpflichtung, sich dem Ziel eines Verteidigungshaushaltes von jeweils $2 \%$ vom Bruttoinlandprodukt innerhalb einer Dekade anzunähern, durch Trendwenden und Steigerung der Haushalte der Mitgliedstaaten vorangetrieben. Dieser Handlungsstrang wurde beim Treffen der Staats- und Regierungschefs, erstmals mit US-Präsident Trump, am 25. Mai 2017 von diesem öffentlich und kontrovers thematisiert; in den Beschlussgremien der Allianz blieb es gleichwohl - im Konsens auch mit den USA - ohne Verschärfungen beim Wortlaut des Defence Investment Pledge von Wales.

\subsection{Perspektiven der neuen NATO}

Das russische Verhalten seit März 2014 hat bei NATO und EU sowie in den Mitgliedstaaten zu einer grundlegend veränderten Beurteilung der Bedrohungslage, einem weitreichenden Paradigmenwechsel, einer ungeahnten Revitalisierung der NATO und zu einer enorm intensivierten NATO-EU-Zusammenarbeit geführt. Dies wird anhand der in der EU beschlossenen wirtschaftlichen Sanktionen gegen Russland und der weitreichenden Beschlüsse der NATO-Gipfel von Wales (2014), Warschau (2016) und Brüssel (Mai 2017) deutlich: In Wales hatte die Allianz als unverzügliches Sofortmaßnahmenpaket - mit verbesserter „Responsiveness“ für Rückversicherung gesorgt. ${ }^{32}$ In Warschau wurde auf der Grundlage sorgfältiger, umfassender Analysen über die Grundprinzipien glaubwürdiger Abschreckung und Verteidigung im 21. Jahrhundert das mittel- und langfristige Konzept zur Stärkung von Abschreckungs- und Bündnisverteidigungsfähigkeit beschlossen. Hierbei hat sich die Allianz mit den Beschlüssen von Wales und Warschau zu Recht auf eine Doppelstrategie in der bewährten Logik der Harmel-Berichts von 1967 geei-

32 Vgl. hierzu Gipfelerklärung von Wales: para 48 (Abschreckung), para 49-50 (Nukleare Dimension), para 51 (Konventionelle Streitkräfte), para 52-64 (Strategische Raketenabwehr), 
nigt, mit der auf der Basis gesicherter Abschreckungs- und Verteidigungsfähigkeit und wiederherzustellender militärischer Stärke zugleich auch der konstruktive Dialog mit Russland gesucht wird. Alle diese Maßnahmen wurden jeweils intensiv in zahllosen Iterationen mit den Hauptstädten ausverhandelt, im Nordatlantikrat der Botschafter beschlossen, durch die Verteidigungsminister, Außenminister und Staats- und Regierungschefs abschließend in Gipfel-relevanten Grundsatzdokumenten gebilligt und zusätzlich in öffentlichen Gipfelerklärungen verankert; seither werden sie unter Hochdruck umgesetzt. Vieles ist bereits in Form gesteigerter Reaktionsfähigkeit und wieder aufgewachsener einsatzbereiter Kräfte und Fähigkeiten vollzogen und wirkt einer möglichen Bedrohung bereits entgegen (z. B. beschleunigte Entscheidungsprozesse, NRF, VJTF, Forward Presence, rotierende Übungspräsen$z e n)$. Dennoch ist der größere Teil der Implementierungsarbeiten mittel- und langfristiger Natur und daher von den Nationen und der NATO als Ganzes unter großen Anstrengungen erst noch zu leisten (u.a. NATO-Kommandostruktur, Großverbände und Verstärkungskräfte; logistische Handlungsfähigkeit SACEURs).

Neben der potentiellen Bedrohung ihrer Mitgliedstaaten durch Russland im Osten wendet sich die Allianz auch den realen Herausforderungen aus dem Süden in Form des Kampfes gegen IS/ISIL/Daesh, und der wachsenden Instabilität vom Maghreb bis zum weiteren Mittleren Osten zu. Im Unterschied zur Bedrohungslage im Osten ist es dem Bündnis allerdings bislang nicht gelungen, umfassende Konzepte zur Bewältigung der deutlich komplexeren Herausforderungen im Süden und zur dortigen Rolle der NATO im Zusammenwirken mit anderen Institutionen zu finden. Es gibt Teilkonzepte zu Einzelaspekten, die aber nicht mit dem konzeptuellen Gesamtpaket zur Stärkung von Abschreckung und Verteidigung mit Blick auf den Osten vergleichbar sind. So lässt sich insbesondere der Abschreckungs-/ Verteidigungs- und zugleich Dialogansatz, der gegenüber Russland erfolgversprechend erscheint, nicht wirksam auf nichtstaatliche Akteure wie IS oder andere islamistische Terrorgruppen, organisierte Kriminalität und die Flüchtlings-/Migrationsproblematik anwenden; auch erhöhte „Responsivness“ von Schnellen Eingreifkräften (NATO Response Force) greift hierbei nur begrenzt. Zum Süden bedarf es gegenwärtig noch konzeptioneller Grundsatzentwürfe. Dieses Missverhältnis zwischen einem weit vorangeschrittenen umfangreichen Konzept zur Wiederherstellung von Abschreckungs- und Verteidigungsfähigkeit gegenüber Russland und andererseits den immer noch vielen offen konzeptionellen Baustellen, wie mit den multiplen und komplexeren Problemen im Süden wirksam umgegangen werden kann, setzt die Kohäsion der Verbündeten unter erheblichen Stress: Auf den Zusammenhalt aller kommt es aber entscheidend an, es gilt ,28 for 28 “ sowohl nach Osten wie nach Süden, anderenfalls würde die Allianz ihre größte Stärke, die Geschlossenheit und Entschlossenheit, einbüßen. Dieses Bemühen wird den bevorstehenden Gipfel in Brüssel im Juli 2018 maßgeblich prägen.

Zugleich hat die jahrzehntelang nur mühsam vorangekommene institutionelle Zusammenarbeit zwischen NATO und EU in den vergangenen 24 Monaten auf Grundlage der beim Gipfel in Warschau von NATO und EU verabschiedeten „Joint Declaration“ Fortschritte erreicht, die einem Quantensprung gleichkommen. ${ }^{33}$ Dies gilt zunächst für das Zusammenwirken des vergleichsweise viel breiteren EU-Spektrums an zivilen (wirtschaftlichen, diplomatischen, administrativen, polizeilichen und anderen) Instrumenten der Krisenvorsorge und -Bewältigung mit den politischen und vorwiegend militärischen Instrumenten der Allianz beim Umgang mit den Herausforderungen im Süden: Projektion von Stabilität, ein altes Grundthema „vernetzter Sicherheit“ im Rahmen des von Deutschland seit langem vertretenen Umfassenden Ansatzes bzw. Comprehensive Approach. Es geht aber ebenso um ein künftig viel engeres und systematisches Zusammenwirken von NATO und EU auch bei der Gewährleitung wirksamer Verteidigungsfähigkeit, denn auch hierbei bedarf es nicht nur gesteigerter militärischer Kräfte, Fähigkeiten und Führungsorganisation auf Seiten der NATO, sondern komplementär auch der Herstellung verbesserter Resilienz insbesondere gegenüber hybriden und Cyber-Attacken. Es geht somit auch um etwas Neues: das Zusammenwirken beider Organisationen mit Blick auf Abschreckung und Verteidigung bei allem, was im Kalten Krieg mit den Planungen und Übungen zur „zivil-militärischen Gesamtverteidigung“34 verbunden war. Sowohl bei der Projektion von Stabilität nach Süden, als auch bei Abschreckung und Verteidigungsfähigkeit nach Osten kommt beiden Organisationen eine je spezifische Hauptrolle zu. Damit ist umfassende, komplementäre institutionelle Zusammenarbeit zwischen NATO und EU wichtiger geworden, als jemals zuvor.

Vor diesem Hintergrund wird die sicherheits- und verteidigungspolitische Agenda, die im Jahr 2018 die Gipfeltreffen der NATO und der EU prägt, weitreichende Entscheidungen verlangen:

- Von der NATO wird beim Gipfel In Brüssel im Juli 2018 eine Bestandsaufnahme der Umsetzungsfortschritte

33 s. hierzu: NATO Statement PR/CP(2016)178, Statement on the Implementation of the Joint Declaration Signed by the President of the European Council, the President of the European Commission, and the Secretary General of NATO, vom 6.Dezember 2016

34 Siehe hierzu die Überlegungen bei Lange 2018. 
und der Konsistenz aller bisher getroffenen Maßnahmen zur substanziell gestärkten Abschreckungs- und Verteidigungsfähigkeit für den Fall eines regionalen Großkonflikts mit Russland erwartet. Dabei kommt es insbesondere auf die Herstellung von Einvernehmen zur renovierten NATO-Kommandostruktur an. Auch ist zu klären, wie weit SACEUR bei seinen Bemühungen gekommen ist, die Fähigkeiten zum zeitgerechten Aufmarsch und zur Verlegung von Großverbänden sowohl mit Blick auf Anfangsoperationen mit NATO Response Force, Enhanced Forward Presence, Kräften der amerikanischen European Reassurance Initiative, als auch mit Blick auf nachfolgend aufwachsende Großverbände als Verstärkungskräfte zu verbessern (,Enabling of SACEURs Area of Responsibility“). Diese umfangreichen Verstärkungskräfte werden vor allem von den USA sowie den großen europäischen Verbündeten gestellt werden müssen, die bei der Bündnisverteidigung gegenüber Russland eine Rolle als Rahmennation für die kleineren Verbündeten leisten können: das sind im Wesentlichen Deutschland und Großbritannien. Frankreich scheidet in dieser Hinsicht aufgrund seines hohen Engagements in Subsahara/Sahelzone aus. Auch wird in Brüssel geprüft werden, wie der Stand bezüglich des Dialogangebots an Russland ist; die aktuelle Entwicklung im Kontext des Russland zugeschriebenen Nervengiftanschlags in Salisbury - als erstem Einsatz eines militärischen Chemiekampfstoffes in Europas seit dem zweiten Weltkrieg, verdeckt ausgeführt auf dem Territorium eines NATO-Verbündeten - erweist sich auch als Gift für die bilateralen und die NATO-Russland-Beziehungen und macht erste ansatzweise Fortschritte in Richtung NATO-Russland-Dialog zunächst wohl wieder zunichte.

- Von der EU werden Entscheidungen erwartet zum Aufbau von verbesserten europäischen VerteidigungsFähigkeiten mit mehr Effizienz in gemeinsamer Entwicklung und Beschaffung. Das Ziel ist hier die Reduzierung bestehender ineffizienter Doppelungen sowie die Steigerung der Effizienz der eingesetzten Verteidigungshaushaltsmittel der europäischen Nationen. Wichtig ist auch die Erhöhung der gesamtstaatlichen und gesellschaftlichen Resilienz, insbesondere im Bereich der Cyber-Sicherheit. Es gilt, das erreichte Momentum bei der NATO-EU-Zusammenarbeit $\mathrm{zu}$ nutzen und diese, nunmehr auch unter substanzieller Beteiligung der Kommission als gewichtigem EUAkteur, konsequent voranzutreiben. ${ }^{35}$

35 Vgl. Helwig 2017.
- Ein wichtiger Bereich ist die Arbeitsteilung zwischen NATO und EU im Bereich Abschreckung und Bündnisverteidigung. Am hochintensiven Ende des Konfliktspektrums ist dies ausschließlich Sache der NATO, diese bedarf aber zunehmend der Unterstützung durch zivile Fähigkeiten der EU im Bereich Hybrid, Cyber und Resilienz.

\section{Was bedeutet das für Deutsch- land und die Bundeswehr?}

Die deutsche Politik steht vor dem Hintergrund der seit 2014 drastisch veränderten Lage - sowohl in der strategischen Richtung Ost als auch der strategischen Richtung Süd - auf der Grundlage der bei den Gipfeln von Wales und Warschau getroffenen Beschlüsse zur Anpassung des Verteidigungsdispositivs der Allianz vor weitreichenden Entscheidungen. ${ }^{36}$ Diese betreffen die Implikationen für die Bundeswehr. Es geht dabei darum, tiefgreifende Veränderungen in der Struktur und der Bewaffnung der Bundeswehr für die kommenden Jahrzehnte und die Steigerung des Finanzrahmens so rational wie möglich zu fundieren. Hierzu wird sich Deutschland mit seinen Land-, Luft-, See- und Cyberstreitkräften, seinem politischen und wirtschaftlichen Gewicht entsprechend, in der Allianz mit einem Anteil von rund 10 Prozent und mindestens gleichauf mit den Beiträgen Großbritanniens und Frankreichs einbringen müssen, um seinen Bündnisverpflichtungen sowie seiner mit dem sog. „Münchner Konsens“ erklärten gesteigerten Verantwortungsbereitschaft zu entsprechen. Aus diesem größten aller europäischen Beiträge erwächst Deutschland zugleich Gestaltungsmacht als europäische Führungsnation. Erst wenn die Bundesrepublik „liefert“, erwächst daraus im Gegenzug eine Führungsrolle sowohl sicherheitspolitisch als auch militärisch, mit der die Diskussion in der Allianz im Sinne der deutschen Interessenlage gestaltet werden kann. Im umgekehrten Falle der Nichteinlösung deutscher Versprechen und dem Zurückbleiben hinter den Erwartungen der Verbündeten steht zu erwarten, dass die Bundesregierung am Ende dem Druck der Verhältnisse doch nachgeben muss, ohne dafür politischen Kredit und Gestaltungsmöglichkeiten zu erhalten.

Für die Bundeswehr bedeutet die Refokussierung der NATO auf die Kernaufgabe Abschreckung, Verteidigung und - auf dieser Grundlage - Dialog mit Russland einen weitreichenden, tiefgreifenden und teuren Paradig-

36 Siehe hierzu den frühen Beitrag zur deutschen Diskussion bei Major 2014 und Major 2015. 
menwechsel des deutschen Verteidigungsdispositivs in nahezu allen Aspekten. Besonders wichtig sind dabei die folgenden:

- Ambitionierte NATO-Fähigkeitenziele für Deutschland: Im Kontext von Landesverteidigung bzw. Bündnisverteidigung hat Deutschland im Rahmen des NATO Verteidigungsplanungszyklus politisch zugesagt, die ihm von der NATO angetragenen Fähigkeitenziele nahezu vollständig zu akzeptieren und zu erfüllen. Dies schließt die Wiederherstellung einsatzbereiter Großverbände ein. Gegenwärtig sind die 2 Divisionen und 6 Brigaden des Heeres zu einem ,force pool“ für Krisenmanagementeinsätze verkommen und können lediglich noch als Organisationselemente, aber nicht mehr als geschlossen einsetzbare Großverbände angesehen werden. Von der Allianz wird wieder auf abgestuft einsatzbereite, aber kampfkräftige, multinationale Großverbände mit wiederhergestellter Fähigkeit zum Gefecht der verbundenen Waffen ab der BrigadeEbene aufwärts gedrängt. Besonders wird von der NATO gefordert, dass Deutschland - über die zugesagten Kräftebeiträge als Rahmennation für die VJTF der $N R F$ sowie den multi-nationalen Gefechtsverband zur verstärkten Vornepräsenz in Litauen hinaus - ein Divisions-Hauptquartier, Divisionstruppen und 2 einsatzfähige Kampfbrigaden kurzfristig bereit hält, um beim Einsatz der enhanced NRF als divisionsgroßer Joint Task Force dieser mit einem nationalen Divisionsäquivalent als Verstärkungs- und Folgekräfte zeitgerecht folgen zu können (vergleichbare Erwartungen der NATO richten sich auch an Großbritannien). Darüber hinaus drängt die NATO langfristig auf Vergrößerung der deutschen Kräftebeiträge um ein drittes Divisionsäquivalent und damit auf einen Heeresumfang von insgesamt 10 Kampfbrigaden in 3 Divisionen (dies allerdings sehr langfristig und bislang nur planerisch).

- Verteidigungsinvestitionsversprechen „2\% Verteidigungsausgaben vom BIP“: Hier steht Deutschland jetzt, fast vier Jahre nach Wales und während der Präsidentschaft Trumps, konkret vor der Entscheidungssituation, entweder das in Wales gegebene und in Warschau bekräftigte Verteidigungsinvestitionsversprechen mit Blick auf das Ziel 2\% vom GDP/ Bruttoinlandsprodukt dilatorisch $\mathrm{zu}$ behandeln und für sich bei der Umsetzung eine Mindererfüllung zu beanspruchen, oder aber den Verteidigungshaushalt in den verbleibenden 8 Jahren zügig sehr stark $\mathrm{zu}$ steigern (von derzeit ca. 40 Milliarden Euro auf die Größenordnung von 60-65 Milliarden Euro in 2024). Diese Steigerung macht allerdings nur Sinn, wenn sie eng an die Erfüllung der NATO Fähigkeitsziele gebun- den bleibt und die Absorptionsfähigkeit für diese enormen Steigerungen (Lieferfähigkeit der Industrie, Mittelabfluss für Projekte) hergestellt worden ist. Die zuletzt veröffentliche vergleichende Übersicht aller Verbündeten ${ }^{37}$ zur Umsetzung des ,2\% vom BIP/20 \% Investitionen"-Ziels zeigt, dass Deutschland (mit $1,24 \% / 13,75 \%$ für 2017) bei diesen politisch hochrelevanten Kriterien - trotz deutlicher Trendumkehr bei seinem Verteidigungshaushalt - bislang kaum voran gekommen ist, noch unter den Median-Werten $(1,30 \% / 19,27 \%)$ liegt und damit - als einzige unter den großen europäische Ländern - nur die untere Hälfte anführt (Portugal, Italien, Slowakei, Kanada, Niederlande, Ungarn, Dänemark, Tschechien, Kroatien, Albanien, Slowenien, Spanien, Belgien). Demgegenüber liegen Großbritannien, Frankreich, Polen, Türkei sowie fast alle exponierten Osteuropäer dicht bei oder über der Zielmarke und deutlich über den Median-Werten. Somit ist die immer noch anhaltende Indifferenz (der alten wie der neuen) Bundesregierung und der sie tragenden Parteien zur Umsetzung des „2\% vom BIP“-Ziels das einzige wirkliche Problem der gegenwärtigen deutschen Bündnispolitik, in einer ansonsten vorzeigbaren und anerkannten Erfolgsbilanz. Diese Indifferenz lässt trotz insgesamt vorbildlichen Verhalten Deutschlands als europäischer Führungsnation neben den USA, Großbritannien und Frankreich immer wieder Zweifel aufkommen, ob Berlin die bisherigen Zusagen wirklich ernst nimmt und nicht nur deklaratorisch mitgetragen hat, um ihre Einlösung letztlich doch zu vermeiden. Von Skeptikern wird vermutet, dass Deutschland die Umsetzung der mittel- und langfristigen Anpassungsmaßnahmen zur Wiederherstellung von Abschreckung und Bündnisverteidigungsfähigkeit zumindest auf die lange Bank zu schieben versucht - zum einen in der Hoffnung auf eine irgendwann durch „Dialog“ herbeigeführte bessere Großwetterlage mit Russland, zum anderen um eine Abrüstungs-affine öffentliche Meinung zu besänftigen. Die Schaffung von Klarheit in dieser Angelegenheit ist entscheidend für die Frage, ob Deutschland es wirklich ernst meint mit der mehrfach erklärten Bereitschaft, seiner gestiegenen Verantwortung gerecht zu werden. Wichtig ist dabei, dass Deutschland wieder seine Rolle als Rückgrat der konventionellen Bündnisverteidigung Europas übernimmt. Zieht Deutschland als politisch und wirtschaftlich gewichtigste europäische Nation hier nicht

37 NATO Press Release PR/CP(2018)16 dtd. 15 March 2018, Defence Expenditure of NATO Countries (2010-2017) 
wie von allen erhofft mit, wird dies weitreichende Auswirkungen auf die Bereitschaft auch aller anderen Nationen haben, ihren Anteil zu liefern.

- Mehrrollenfähigkeit: Die Truppenkörper und Verbände der Bundeswehr müssen in viel stärkerem Umfang als bisher sowohl für wirksames Krisenmanagement im Rahmen kleinerer und mittlerer Stabilisierungsoperationen ausgebildet, ausgerüstet und bewaffnet sein, als auch zugleich nach kurzer oder sehr kurzer Vorbereitung als kampfkräftige Manöverelemente in einem hochintensiven Konflikt am oberen Ende im Rahmen einer „MJO plus“ zur kollektiven Bündnisverteidigung einsetzbar und verlegefähig sein. Diese Anforderung, beides zugleich zu können, kann von den gegenwärtigen deutschen Streitkräften in den jetzigen Strukturen und mit der jetzigen Haushaltsausstattung nicht geleistet werden, kommt aber unausweichlich auf sie $\mathrm{zu}$.

- Rahmenneutralität: Neben der Mehrrollenfähigkeit der Verbände der Bundeswehr gilt es, grundsätzlich eine multiple Rahmenfähigkeit bzw. Rahmenneutralitität herzustellen. Das bedeutet, die Verbände, Kräfte, Fähigkeiten und Mittel der Bundeswehr müssen sowohl im Rahmen der NATO, als auch der EU, oder der VN oder von Koalitionsoperationen einsetzbar und führbar sein - eine Forderung, die in den letzten Jahren bereits weitgehend zur Realität geworden ist, aber weiterhin der Optimierung bedarf.

- Rahmennationenkonzept: Für die Großverbände der Bundeswehr gilt der Grundansatz, dass diese künftig grundsätzlich nicht mehr national, sondern multinational-europäisch im Verbund mit den Deutschland umgebenden Nachbarn im „Rahmennationenkonzept" entwickelt werden sollen. Die Idee hierbei ist, den notwendigen Schritt zur Wiederherstellung einsatzbereiter deutscher Großverbände als Folgekräfte und Verstärkungen mit Blick auf kollektive Bündnisverteidigung mit dem Imperativ zu verbinden, integrierter und leistungsfähiger europäischer Streitkräfte zur Stärkung des europäischen Pfeilers in der Allianz und gleichzeitig zur Stärkung der Handlungsfähigkeit der EU. Schlüsselaktivität hierfür ist das von Deutschland angeführte „Framework Nation Grouping on Capabilty Development and Larger Formations“. Diese in Wales entstandene, mittlerweile zwei Drittel der europäischen Verbündeten und auch erste Partnernationen wie Schweden und Finnland umfassende Gruppe mit Deutschland als Führungs- und Rahmennation hat sich auf der Grundlage des NATO Framework Nation Concept in Absprache mit der Allianz das Ziel gesetzt, zum einen in den erkannten prioritären
Fähigkeitenbereichen bestehende Defizite zu schließen und zum anderen zwei bis drei einsatzbereite Heeresdivisionen (larger formations) gemeinsam verfügbar zu machen.

Vom Erfolg dieser von Deutschland initiierten engen Zusammenarbeit hängt $a b$, ob es gelingt, unter den europäischen Verbündeten insgesamt zu einem deutlich verbesserten „Output“ an militärischer Wirkung und Effizienz zu kommen. Für die bessere Ausbalancierung zur transatlantischen und innereuropäischen Lastenteilung ist dies von großer Bedeutung: Während das 2\%/20\%-Ziel des Defence Investment Pledge auf der Input-Seite vorrangig darauf abzielt, das bestehende 70:30-Ungleichgewicht zwischen den USA und Europa ${ }^{38}$ in Richtung 50:50 auszubalancieren, ist die Diskrepanz auf der Output-Seite, was Kampfkraft und Fähigkeiten angeht, noch krasser und liegt geschätzt (nach amerikanischen, aber auch nach Angaben der EU) bei ca. 85:15. Wenn es - komplementär zur Behebung des Ungleichgewichts auf der Input-Seite mittels des Defence Investment Pledge (2\%/20\%) - gelänge, dieses Mal (im Unterschied zu den früheren Initiativen „Smart Defence“ und Pooling and Sharing") mit dem systematischer, tiefgreifender und nachhaltiger ansetzenden Framework Nation Concept dieses Missverhältnis in Richtung 70:30 oder sogar 50:50 auch auf der Output-Seite zu verbessern, wäre dies nicht nur die Lösung des Dauerbrenners „faire transatlantische Lastenteilung“. Es wäre auch ein höchst substanzieller Fortschritt hin zu kampfkräftigeren, interoperablen Streitkräften der europäischen Nationen, die zwar noch lange keine „Europa Armee“ ergeben, der EU aber dennoch eine größere eigene sicherheitspolitische Handlungsfähigkeit eröffnen würden. Ob dieses Ziel erreicht wird, hängt von keiner Nation so sehr ab wie von Deutschland, und es entscheidet sich in erster Linie an der Höhe des deutschen Verteidigungshaushaltes und dem davon finanzierten Rahmen, den Deutschland als Führungsnation seinen mit ihm gehenden europäischen Kooperationspartnern für deren Beiträge zu bieten vermag.

\section{Fazit und Ausblick}

Die Allianz hat ab März 2014 unerwartete strategische Extremsituationen (Russland im Osten, ISIL/DAESH im

38 Die USA tragen gegenwärtig mit ca. 70 \% den Löwenanteil der Gesamtsumme aller Verteidigungsausgaben der NATO-Mitgliedstaaten, während die europäischen Verbündeten und Kanada zusammen die übrigen $30 \%$ aufbringen. 
Süden) zu bewältigen gehabt - bei zugleich fortlaufendem „Normalbetrieb“ in Form von vielfältigem Krisenmanagements mittleren und niedrigen Anspruchsniveaus an der südlichen Peripherie (u.a. ISAF/RSM-Missionen in Afghanistan, KFOR im Kosovo) sowie bei der Kooperativen Sicherheit mit Partnernationen und -Organisationen mit globaler Reichweite. Sie hat dabei Entschlossenheit, Geschlossenheit und Handlungsfähigkeit unter Beweis stellen und aus den Herausforderungen lernen müssen.

Dies hat $\mathrm{zu}$ einem enormen Revitalisierungsschub und einer umfassenden Anpassung - politisch, militärisch und institutionell - an eine von Grund auf veränderte sicherheitspolitische Lage geführt. Mit den dabei getroffenen politischen Grundsatzentscheidungen sind die Weichen in Richtung eines grundlegend veränderten Verteidigungsdispositiv (Defence Posture) der Allianz und damit zugleich auch entsprechend veränderter Verteidigungsdispositive aller ihrer Mitgliedstaaten - einschließlich Deutschlands und seiner Bundeswehr sowie seines zivilen und militärischen GesamtverteidigungsKonzepts - gestellt worden.

Deutschland hat die Veränderungen seit 2014 nicht nur mitgetragen, sondern in führender Rolle konstruktiv mitgestaltet und mit vielfältigen Beiträgen an Verteidigungsinvestitionen, Fähigkeiten und Kräftebeiträgen zur Steigerung der Reaktionsfähigkeit (Wales) und der Stärkung des Abschreckungs- und Verteidigungsdispositivs (Warschau) in alle strategischen Richtungen beispielgebend beigetragen. Auch wenn viele Impulse von den kollektiven Entscheidungen der NATO zu 28 in Brüssel ausgegangen sein mögen, so war in Berlin mit dem erklärten Willen zu „mehr Verantwortung“ in „führender Rolle aus der Mitte“ doch zumindest die grundsätzliche Akzeptanz und Bereitschaft gegeben, den Impulsen zu folgen und das Nötige zu leisten ${ }^{39}$.

Insgesamt sind die konzeptionellen Arbeiten zum Thema Abschreckung und Verteidigung weitgehend geleistet und erscheint ihre bereits begonnene, rasch fortschreitende Umsetzung geeignet, Russland wirksam davon abzuhalten, Konflikte mit den Mitgliedstaaten von NATO und EU militärisch zu lösen.

Es gilt darüber hinaus sich darauf einzustellen, dass Geopolitik wieder zurück ist und der „Westen“, und in ihm Europa und damit auch Deutschland, es sicherheitspoli-

39 Siehe hierzu als offene Quellen insbesondere die Beiträge zur öffentlichen Diskussion des deutschen Ständigen Vertreters bei der NATO, Botschafter Dr. Hans-Dieter Lucas, in Vorträgen und Namensartikeln seit 2015 (Lucas 2016, Lucas 2017a, Lucas 2017b). Ebenso die Namensartikel vom Leiter der Planungsabteilung des BMVg, GenLt Erhard Bühler (z. B. Bühler 2016), und dem InspH, GenLt Vollmer (Vollmer 2017). tisch mit einer konkurrierenden Großmachtkonstellation zu tun haben: kurz- und mittelfristig offensichtlich Russland, längerfristig wohl auch China. Hieran angelehnt bildet sich möglicherweise ein Lager mit weiteren Kontrahenten, die sich bereits als gefährliche „spoiler“ der westlichen regelbasierten Ordnung erwiesen haben: Nordkorea, Iran, Syrien. Ob sich aus der Entfremdung der Türkei die Gefahr eines Wechsels in dieses Lager ergibt, ist eine offene Frage, Anzeichen dafür lassen sich nicht mehr übersehen. Gegen eine derartige Gruppierung alter und neuer Mächte, die allesamt staatliche Akteure sind, vermag das alte Harmel-Konzept dem Prinzip nach genauso wirkungsvoll zu greifen wie in der Ära des Ost-West-Konflikts mit der Sowjetunion: $\mathrm{S}=\mathrm{V}+\mathrm{E}$. Sollte eine solche Konstellation entstehen, wäre die Bedrohung weniger konkret und eher potentieller Art, die globale Reichweite des Konflikts wäre - unter Einschluss Chinas und damit Asien-Pazifischen Raums - größer. Es gäbe nicht eine einzige gegnerische Hauptstadt (Moskau), um das herum sich SatellitenStaaten gruppieren, sondern der Westen hätte es mit einer durchaus heterogenen und fragilen Koalition zu tun, die sich nur in der Ablehnung westlichen Werte, Regeln und Ordnungsvorstellungen einig ist. Auch sind die militärischen Kräfteverhältnisse quantitativ und qualitativ noch immer insgesamt günstiger für den Westen, allerdings mit abnehmender Tendenz.

Es sprich deshalb viel dafür, sich bei der Wiederherstellung von Abschreckung und Verteidigung und der Wiedergewinnung dafür nötiger militärischer Kräfte und Fähigkeiten kurz- und mittelfristig wieder zu einer bedrohungsorientierten Verteidigungsplanung überzugehen, die sich am russischen Potential ausrichtet und zugleich langfristig auch das rasch aufwachsenden chinesische militärische Potential mit auf den Schirm nimmt. Zugleich gilt es, mit den vorhandenen und den künftigen Kräften und Fähigkeiten, die primär wieder auf Abschreckungsund Verteidigungsfähigkeit am hochintensiven Ende ausgerichtet sein müssen, die vielfältigen und voraussichtlich noch zunehmenden Einsätze zur Krisenprävention und -Bewältigung im Süden Europas zu leisten (,within means and capabilities“). „Abwärts“ ist dies machbar; anders herum - „aufwärts“ - besteht eine solche Option nicht: Mit den Kräften und Fähigkeiten eines insgesamt weiterhin lediglich auf Krisenmanagement ausgerichteten Verteidigungsdispositivs kurzfristig und unvorbereitet auch in hochintensiver Konfliktaustragung der Ebene „Major Joint Operation Plus" bestehen zu wollen und daraus abschreckende Wirkung zu erhoffen, wäre das sicherste „Rezept für Desaster“: es würde bedeuten, gegenüber einem risikobereiten Gegner im Testfall vor die Wahl gestellt zu werden, seine militärischen „fait accomplies“ hinzuneh- 
men oder in einem Krieg mit hohen Opfern absehbar zu unterliegen.

Für die deutschen Streitkräfte folgt daraus, dass Planung sich auf Mehrrollenfähigkeit, Rahmenneutralität, langfristig stetigen Aufwuchs des Verteidigungshaushalts Richtung 2\% vom BIP und 20\% Investitionen bis 2024 basieren sollte. Die Umsetzung der akzeptierten NATOKräfte- und Fähigkeitenziele für Deutschland erfordert für Mitte der 2020er Jahre (also ca. 2024/2025, 10 Jahre nach Wales) einen jährlichen Verteidigungshaushalt in Höhe von etwa 60 bis 65 Mrd. Euro - dies läge dicht an dem Richtwert von $2 \%$. Einerlei, ob die Politik nun primär die Erfüllung der zugesagten NATO-Fähigkeitenziele oder des 2\%-Ziels als Maßgabe gelten lässt, der deutsche Verteidigungshaushalt bedarf in beiden Fällen einer stetigen jährlichen Steigerung um 3 bis 4 Mrd. Euro bis Mitte der 2020er Jahre. Ein solcher zusätzlicher Mittelzufluss pro Haushaltsjahr muss in den mittlerweile hoffentlich reparierten Forschungs-, Entwicklungs- und Beschaffungsprozessen absorbierbar sein, anderenfalls griffe weiterhin das „Totschlagsargument“, BMVg und Bundeswehr könnten einen jährlich substanziell steigenden Verteidigungshaushalt ohnehin nicht sinnvoll in leistungsgesteigerte Kräfte und Fähigkeiten umsetzen. Das andere, in der deutschen Diskussion oft kolportierte Argument, ein Aufstieg Deutschlands zur stärksten konventionellen Militärmacht sei von Paris, London und Warschau nicht wirklich gewollt, hat sich mit dem anhaltenden Drängen nicht nur Washingtons, sondern ebenso dieser europäischen Verbündeten, die ein ganz offensichtliches und plausibles gemeinsames Interesse an Entlastung durch Deutschland und seine Bundeswehr haben - Frankreich mit Blick auf die SahelZone; Großbritannien und Polen mit Blick Bündnisverteidigung im Osten - schon seit längerem erledigt.

Abschließend bleibt festzuhalten, dass Deutschland bündnispolitisch seit 2014 fast alles richtig gemacht hat, mit Ausnahme seiner im Wahlkampf 2017 offensichtlich gewordenen Indifferenz zum 2\%-Ziel. Nun kommt es darauf an, auch bei der Umsetzung der mitgestalteten Beschlüsse weiterhin mit gutem Beispiel voranzugehen, um glaubwürdige Abschreckung und Verteidigungsfähigkeit, nicht zuletzt auch als Grundlage für den Erfolg von Bemühen um Entspannung mit Russland, wiederherzustellen. Nur wenn dies im engen transatlantischen europäischen Verbund gelingt, werden wir die bestehende, unseren Werten und Interessen entsprechende, regelbasierte internationale Ordnung gegenüber den Gegnern dieser Ordnung auf Dauer bewahren können.

\section{Literatur}

Adomeit, Hannes (2017): Innenpolitische Determinanten der Putinschen Außenpolitik, SIRIUS 1 (1) 33-52.

Bruns, Sebastian/Kirchberger, Sarah (2017): Machtprojektion ins NATO-Meer: Die Marine der VR China in der Ostsee. SIRIUS, 1 (4), 379-387.

Bühler, Erhard (2015): Gestaltungsmöglichkeiten ausschöpfen, in: Info Brief Heer, hrsg. vom Förderkreis Deutsches Heer, 5.

Glatz, Rainer L./Zapfe, Martin (2017): Ambitionierte Rahmennation: Deutschland in der NATO. Die Fähigkeitenplanung der Bundeswehr und das „Framework Nations Concept“. Berlin: SWP Aktuell 62.

Helwig, Niklas (2017): Neue Aufgaben für die Zusammenarbeit zwischen EU und NATO. Eine inklusive EU-Verteidigungspolitik setzt eine enge Kooperation mit der NATO voraus. Berlin: SWP Aktuell 80.

Kamp, Karl-Heinz (2017a): Grundzüge einer neuen NATO-Strategie, SIRIUS 1 (1), 25-32.

Kamp, Karl-Heinz (2017b): Eine neue Nuklearausrichtung der NATO, SIRIUS 1 (4) 359-366.

Krause, Joachim (2017): Die neue Zeitenwende in den internationalen Beziehungen - Konsequenzen für die deutsche und europäische Außenpolitik, SIRIUS 1 (1), 3-24.

Lange, Philipp (2018): Gesamtverteidigung 2.0. Wie sollte Landesund Bündnisverteidigung gesamtstaatlich umgesetzt werden? Berlin: BAKS Arbeitspapier Sicherheitspolitik, Nr. 2.

Lucas, Hans-Dieter (2016): NATO zwischen Wales und Warschau. Anpassung heißt nicht, alles über Bord zu werfen, Die Bundeswehr, hrsg. vom Deutschen Bundeswehrverband, Mai. Lucas, Hans-Dieter (2017a):The North Atlantic Alliance in an Age of Uncertainties - a European Perspective, Berlinpolicyjournal. com, 17. 1. 2017

Lucas, Hans-Dieter (2017b): Useful Principles. The Hamel Report, released at the height of the Cold War, laid out a blueprint for NATO's role in Europe and its relationship with Russia. The approach is useful today, Berlinpolicyjournal.com, 14. 12. 2017.

Major, Claudia (2014): Der NATO-Gipfel 2014 im Schatten der Ukraine-Krise. Berlin; SWP Aktuell 51/2014.

Major, Claudia (2015): Die Strategische Anpassung der NATO Deutschland ist das Rückgrat für die militärische Neuaufstellung der Allianz. Berlin; SWP-Aktuell 20.

Meier, Ernst-Christoph/Roßmanith, Richard / Schäfer, Heinz-Uwe, Hrsg. (2003): Wörterbuch zur Sicherheitspolitik - Deutschland in einem veränderten internationalen Umfeld, 5. Auflage, Koblenz; Mittler-Verlag.

Meier, Ernst-Christoph/Hannemann, Andreas / Meyer zum Felde, Rainer, Hrsg. (2012): Wörterbuch zur Sicherheitspolitik Deutschland in einem veränderten internationalen Umfeld, 8. Auflage, Koblenz; Mittler-Verlag 2012 (ISBN 978-3-81320933-4)

Menkiszak, Marek (2017): Herausforderung Russland. Worin sie besteht und wie darauf zu reagieren ist. Berlin: BAKS Arbeitspapier Sicherheitspolitik, Nr. 27.

Vollmer, Jörg (2017): Keine Zeit sich auszuruhen! Info Brief Heer. 1, Februar. 